\title{
Drug development's dark side
}

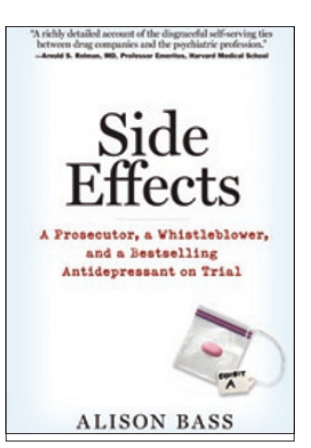

\section{Side Effects: A Prosecutor, a Whistleblower, and a Bestselling Antidepressant on Trial}

\section{Alison Bass}

Algonquin Books, 2008

260 pp., hardcover, $\$ 24.95$

ISBN: 1565125533

\section{Reviewed by Joseph T Coyle}

In the late 1980s, on the basis of psychoanalytic theory, child psychiatrists generally believed that children were incapable of becoming depressed. In spite of this, children as young as ten years old were hospitalized because of symptoms of severe depression. Epidemiological studies indicated that suicide was the third most common cause of death among adolescents, and "psychological autopsies" revealed that the vast majority were suffering from untreated depression. Because these depressed children often satisfied the diagnostic criteria for major depressive disorder, clinicians assumed that they should respond to the same treatment as adults. The new class of 'benign' serotonin-specific reuptake inhibitors (SSRIs) seemed to be a particularly attractive treatment. I provide this background as context to Alison Bass's excellent book Side Effects, about how good intentions to effectively treat depressed patients can be subverted by an increasingly market-driven pharmaceutical industry.

The narrative begins with an academic psychiatrist affiliated with a prominent medical school. In 1990, he published an article describing six pediatric patients who, when receiving fluoxetine (Prozac, Eli Lilly), an SSRI, became agitated and suicidal and engaged in self-injurious behavior. I use the term 'narrative' because Bass brings to life dry policy issues through her vivid portrayal of three protagonists: the psychiatrist, a lawyer involved in litigation over SSRI use in children and a mother of a child with a severe mood disorder. Bass follows these individuals over a 15-year period as their paths intersect in unexpected ways and shows how their persistent efforts ultimately led to major changes in the way the pharmaceutical industry markets new drugs.

After the psychiatrist's article was published, he was criticized by his colleagues because of the perception that he was discrediting an effective treatment for children with depression. The personal toll from questioning the safety of the lucrative new antidepressants ultimately caused him to move into a different area of clinical research, and his marriage unraveled when his physician wife was hired by the same company that marketed the offending SSRI.

The second protagonist is a woman whose adoptive daughter had a severe form of bipolar disorder. Motivated by her daughter's illness, she took a position as an administrative assistant in a university depart-

Joseph T. Coyle is the Eben S. Draper Professor of Psychiatry and Neuroscience at Harvard Medical School, Belmont, Massachusetts, USA.

e-mail: joseph_coyle@mac.com ment of psychiatry. She uncovered questionable reimbursements to the department from the state that she disclosed to the press. For example, the chairman of the department had substantial undisclosed financial relationships with the pharmaceutical industry.

The third protagonist, the lawyer, was hired to work in the Office of the New York Attorney General. She succeeded in compelling a major drug company to disclose the results of all its clinical trials in children with the SSRI paroxetine. The results demonstrated that not only was the drug ineffective in treating depression in children, but also it significantly increased the risk for agitation and suicidal thoughts in comparison to placebo treatment.

Highlighted by the book is an extensive web of unrecognized financial conflicts of interest. Many of the critics of the psychiatrist received funding from industry. The US Food and Drug Administration (FDA) had come under increasing sway of industry as 'user fees' now dominated its budget, and pressure was exerted to ensure rapid approval of new drugs. Patient advocacy groups receiving donations from industry encouraged their members to participate in industry-sponsored clinical trials. The very investigators who had received hundreds of thousands of dollars to conduct clinical trials also served as 'key opinion leaders' and were lavishly compensated for giving lectures touting the very same drugs.

As a consequence of the disclosures about paroxetine, the data from all of the clinical trials of SSRIs in children and adolescents were reexamined by the FDA. In 2004, the FDA issued a 'black box' warning that antidepressants can increase suicidality (thoughts of self-harm) in the pediatric population. Although suicidality was markedly higher in children and adolescents receiving SSRIs than those receiving placebo (a difference that disappears in adults), there were no more actual suicides in the SSRI group. Presumably because of the black box warning, the number of youth prescribed antidepressants has decreased substantially since 2004, especially among those treated by primary care physicians.

The lack of transparency for drug development that became apparent to Congress with the SSRI disclosures has caused a radical change in how the pharmaceutical industry develops new drugs. First, industry as well as academic investigators must prospectively register their clinical trials in the public domain (for example, on http://www.clinicaltrials.gov, a publicly accessible web site that is maintained by the US National Library of Medicine), presenting the design, number of subjects and primary outcomes. The major scientific journals will not accept articles on the results of clinical trials that have not been prospectively registered. This prevents multiple publications of components of one trial, which can give the misimpression of multiple positive studies. Registration also prevents the suppression of negative or failed clinical trials so that the lack of reproducibility of findings is evident. Medical schools are now setting up explicit limits on the amount of money that an investigator can receive from a company and still be able to conduct a clinical trial.

Sunlight is the best disinfectant. Thanks to the persistence of the individuals described so eloquently by Alison Bass in Side Effects, much more light is illuminating the dark side of drug development.

\section{COMPETING INTERESTS STATEMENT}

The author declares competing financial interests: details accompany the full-text HTML version of the paper at http://www.nature.com/naturemedicine/. 